\title{
Comparison of free amino acids, antioxidants, soluble phenolic acids, cytotoxicity and immunomodulation of fermented mung bean and soybean
}

\begin{abstract}
BACKGROUND: Mung bean and soybean have been individually reported previously to have antioxidant, cytotoxic and immunomodulatory effects, while fermentation is a wellknown process to enhance the bioactive compounds that contribute to higher antioxidant, cytotoxic and immunomodulation effects. In this study, the free amino acids profile, soluble phenolic acids content, antioxidants, cytotoxic and immunomodulatory effects of fermented and non-fermented mung bean and soybean were compared. RESULTS: Fermented mung bean was recorded to have the highest level of free amino acids, soluble phenolic acids (especially protocatechuic acid) and antioxidant activities among all the tested products. Both fermented mung bean and soybean possessed cytotoxicity activities against breast cancer MCF-7 cells by arresting the G0/G1 phase followed by apoptosis. Moreover, fermented mung bean and soybean also induced splenocyte proliferation and enhanced the levels of serum interleukin-2 and interferon-כ. CONCLUSION: Augmented amounts of free amino acids and phenolic acids content after fermentation enhanced the antioxidants, cytotoxicity and immunomodulation effects of mung bean and soybean. More specifically, fermented mung bean showed the best effects among all the tested products. This study revealed the potential of fermented mung bean and soybean as functional foods for maintenance of good health.
\end{abstract}

Keyword: Fermentation; Immunomodulation; Mung bean; Protocatechuic acid; Soybean 